\title{
Breaking projective chaos synchronization secure communication using filtering and generalized synchronization
}

\author{
G. Álvarez ${ }^{\mathrm{a}, *}$, Shujun Li ${ }^{\mathrm{b}}$, F. Montoya ${ }^{\mathrm{a}}$, G. Pastor $^{\mathrm{a}}$ and \\ M. Romera ${ }^{a}$ \\ ${ }^{a}$ Instituto de Física Aplicada, Consejo Superior de Investigaciones Científicas, \\ Serrano 144-28006 Madrid, Spain \\ ${ }^{\mathrm{b}}$ Department of Electronic Engineering, City University of Hong Kong, Kowloon, \\ Hong Kong SAR, China
}

\begin{abstract}
This paper describes the security weaknesses of a recently proposed secure communication method based on chaotic masking using projective synchronization of two chaotic systems. We show that the system is insecure and how to break it in two different ways, by high-pass filtering and by generalized synchronization.
\end{abstract}

\section{Introduction}

In recent years, a considerable effort has been devoted to extend the chaotic communication applications to the field of secure communications. The possibility of synchronization of two coupled chaotic systems was first shown by Pecora and Carrol [1-3] and opened the possibility of using the signals generated by chaotic systems as carriers for analog and digital communications. This discovery soon aroused great interest as a potential means for secure communications [4]. Accordingly, a great number of cryptosystems based on chaos have been proposed [5-9], some of them fundamentally flawed by a lack of robustness and security [10-16].

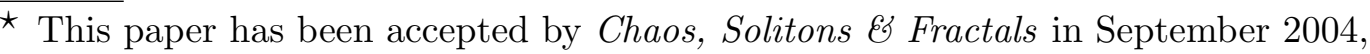
and has been available online at http://dx.doi.org/10.1016/j.chaos.2004.09. 038.

* Corresponding author: Email: gonzalo@iec.csic.es 
Projective synchronization (PS) is an interesting phenomena firstly described by Mainieri and Rehacek [17], it consists in the synchronization of two partially linear coupled chaotic systems, master and slave, in which the amplitude of the slave system is a scalar multiple, called scaling factor, of that of the master system in the phase space. The original study was restricted to threedimensional partially linear systems. Later $\mathrm{Xu}$ and $\mathrm{Li}$ [18] showed that PS could be extended to general classes of chaotic systems without partial linearity, by means of the feedback control of the slave system; they illustrated the applicability to Lorenz, Chua and the hyperchaotic Rössler systems.

In a recent paper $\mathrm{Li}$ and $\mathrm{Xu}$ [19] proposed a secure communication scheme based on PS chaotic masking. The authors claimed that the unpredictability of the scaling factor of the PS can additionally enhance the security of communications. Furthermore, the authors proposed the use of an invertible function $F$, in such a way that the transmitted ciphertext signal will be $U(t)=x_{1}+F\left[x_{1}, y_{1}, z_{1}, m(t)\right]$, where $x_{1}, y_{1}$ and $z_{1}$ are the variables of a three-dimensional chaotic system and $m(t)$ is the plaintext (which in their paper is assumed to be a sound signal). They claimed that the security of the information can also be guaranteed because the function $F$ could be arbitrarily chosen. They illustrated the feasibility of the scheme with two examples, based on the Lorenz and the hyperchaotic Rössler systems, respecitvely.

In this article we show that the proposed cryptosystem is insecure and how to break it in two different ways, by high-pass filtering and by generalized synchronization, for both examples based on the Lorenz and the hyperchaotic Rössler systems.

In [19], the first example is based on the following Lorenz system:

$$
\begin{aligned}
& \dot{x_{1}}=\sigma\left(y_{1}-x_{1}\right), \\
& \dot{y_{1}}=(\mu-z) x_{1}-y_{1}, \\
& \dot{z_{1}}=x_{1} y_{1}-\rho z_{1} .
\end{aligned}
$$

with parameter values $\{\sigma, \mu, \rho\}=\{10,60,8 / 3\}$. The transmitted signals from the sender to the receiver end are the shared scalar variable $z_{1}$ and the ciphertext $U(t)=x_{1}+F\left[x_{1}, y_{1}, z_{1}, m(t)\right]$, where the function $F$ was specified as $F\left[x_{1}, y_{1}, z_{1}, m(t)\right]=y_{1}+m(t)$.

The second example is based on the hyperchaotic Rössler system defined by the authors as:

$$
\begin{aligned}
\dot{x_{1}} & =-y_{1}-z_{1}, \\
\dot{y_{1}} & =x_{1}+a y_{1}+w_{1}, \\
\dot{z_{1}} & =b+x_{1} z_{1}, \\
\dot{w_{1}} & =c z_{1}+d w_{1} .
\end{aligned}
$$


with parameter values $\{a, b, c, d\}=\{0.25,3,-0.5,0.05\}$. In this example, the transmitted signals from the sender to the receiver end are the shared scalar variable $w_{1}$ and the ciphertext $U(t)=x_{1}+F\left[x_{1}, y_{1}, z_{1}, w_{1}, m(t)\right]$, where $F$ was specified as $F\left[x_{1}, y_{1}, z_{1}, w_{1}, m(t)\right]=y_{1}+m(t)$.

\section{Loose system key specification}

Although the authors seem to base the security of its communication system on the chaotic behavior of the output of a chaotic or hyperchaotic non-linear system, no analysis of security was included. Instead, an unproved assertion saying that "the security of information can be guaranteed" was given in the conclusion.

The first issue to be considered is the key of the system. A cryptosystem cannot exist without a key, otherwise, it might be considered as a coding system, but never regarded as a secure system. In [19] it is not considered whether there should be a key in the proposed system, what it should consist of, what the available key space would be, and how it would be managed.

When cryptanalyzing a cryptosystem, the general assumption made is that the cryptanalyst knows exactly the design and working of the cryptosystem under study, i.e., he knows every detail about the ciphering algorithm, except the secret key. This is an evident requirement in today's secure communications systems, usually referred to as Kerckhoffs' principle [20].

In [19] it was stated that the arbitrary selection of the function $F$ will warrant the information security. But according to the Kerckhoffs' principle, the function $F$ must be publicly known and may not be considered part of the key. At most, its structure could contain some factors or constants whose values can play the role of secret key; but, unfortunately, the authors of [19] have not considered such possibility, nor which conditions the function $F$ might satisfy, nor how many usable functions there are, nor how much they can contribute to the system security. Much care must be exercised when selecting the function $F$. Otherwise, choosing different functions might create different ciphertexts, that can be decrypted using the same algorithm though, as shown in Sec. 4.

\section{$3 \quad$ Inefficiency as a masking system}

It is supposed that chaotic masking is an adequate means for secure transmission, because chaotic systems present some properties as sensitive dependence on parameters and initial conditions, ergodicity, mixing, and dense periodic 

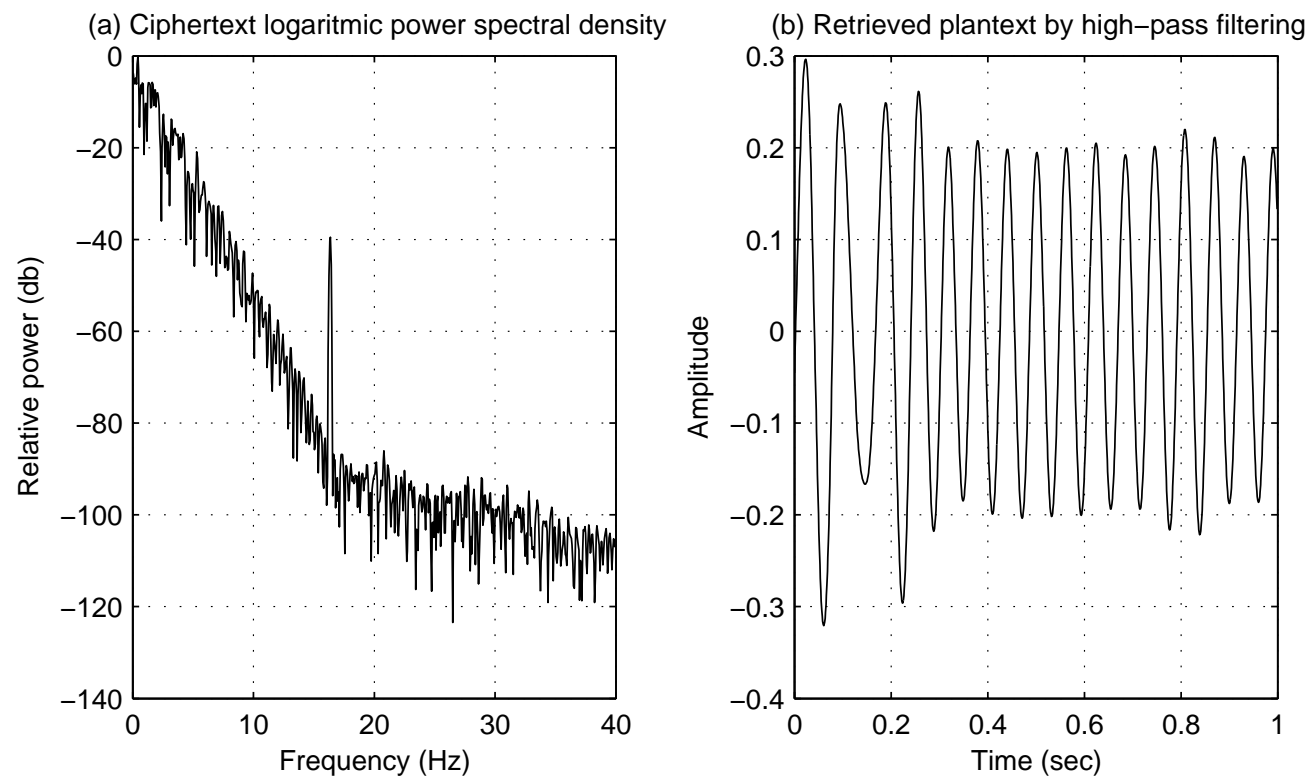

Fig. 1. Encrypted transmission of a plaintext of amplitude 0.2 and frequency 16.352 $\mathrm{Hz}$, by masking with the Lorenz system described in [19]: (a) logarithmic power spectrum of the ciphertext; (b) retrieved plaintext by high-pass filtering of the ciphertext.

points. These properties make them similar to pseudorandom noise [21], which has been used traditionally as a masking signal for cryptographic purposes. A fundamental requirement of the pseudorandom noise used in cryptography is that its spectrum should be infinitely broad, flat and of much higher power density than the signal to be concealed. In other words, the plaintext power spectrum should be effectively buried into the pseudorandom noise power spectrum. The cryptosystem proposed in [19] does not satisfy this condition. On the contrary, the spectrum of the signal generated by the Lorenz oscillator is of narrow band, decaying very fast with increasing frequency, showing a power density much lower than the plaintext at plaintext frequencies.

In [19] the sound of a water flow was used as the plaintext message $m(t)$, but no details are given about its waveform or power spectrum. From [19, Fig. 2] it can be appreciated that its amplitude is roughly 0.2 . In our simulation we have used, instead, a well defined plaintext signal $m(t)=\sin (2 \pi 16.352 t)$, which corresponds to a pure tone sound of $16.352 \mathrm{~Hz}$, which is the lowest note generated by a musical instrument, the $\mathrm{C}_{0}$ of a $32 \mathrm{ft}$ pipe of a pipe-organ $[22,23]$ and with the same peak amplitude of [19, Fig. 2], namely 0.2 .

Figures 1(a) and 2(a) illustrate the logarithmic power spectra of the ciphertext when the Lorenz attractor and the hyperchaotic Rössler attractor are used as the chaotic system, respectively, with the same parameter values previously described. 

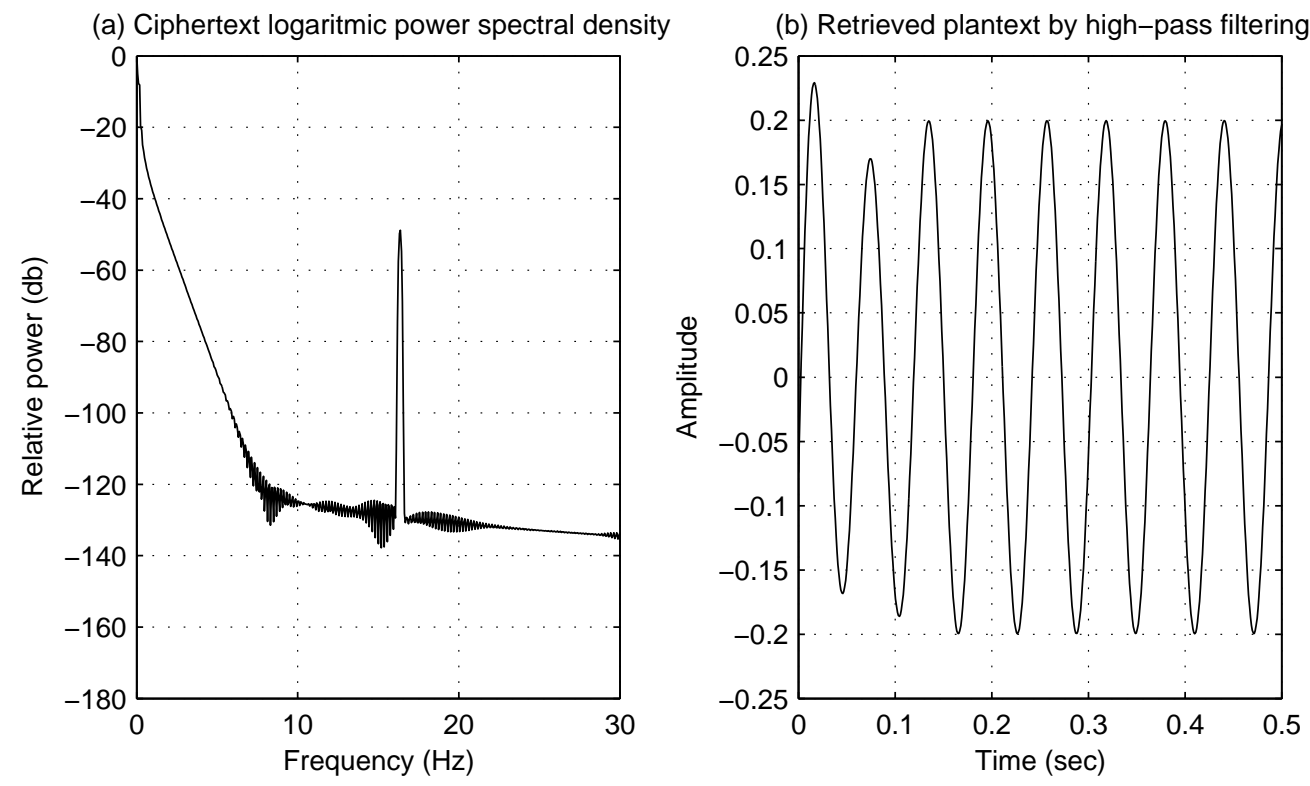

Fig. 2. Encrypted transmission of a plaintext of amplitude 0.2 and frequency 16.352 $\mathrm{Hz}$, by masking with the hyperchaotic Rössler system described in [19]: (a) logarithmic power spectrum of the ciphertext; (b) retrieved plaintext by high-pass filtering of the ciphertext.

It can be seen that in both examples the plaintext signal clearly emerges at $16.352 \mathrm{~Hz}$ over the background noise created by the Lorenz and hyperchaotic Rössler oscillators, with a power of $-40 \mathrm{db}$ and $-50 \mathrm{db}$, respectively, relative to the maximum power of the ciphertext spectrum, while the power density of the ciphertext, at neighboring frequencies, falls below $-80 \mathrm{db}$ and $-125 \mathrm{db}$, respectively.

To recover the plaintext we did not use a chaotic receiver, instead the ciphertext was high-pass filtered. The procedure is illustrated in Fig. 1(b) and 2(b). The filter employed was a finite impulse response one. To avoid phase nonlinearities and distortion, it was constructed with a 512-coefficient Hamming window, with a cutoff frequency of $13 \mathrm{~Hz}$. The result is a good estimation of the plaintext after a short initial transient of approximately 0.3 seconds for the Lorenz system. Note that this is the hardest case an attacker can face from the point of view of plaintext frequency, because for higher sound frequencies the spectrum of the background noise created by the Lorenz oscillator is even lower. While for the hyperchaotic Rössler system the result is a perfect recovery after a short initial transient of approximately 0.1 seconds. 


\section{Generalized synchronization attack}

The former attack method works only for plaintext frequencies higher than the $13 \mathrm{~Hz}$ cut-off frequency of the high-pass filter employed. For very low plaintext frequencies the noise created by the chaotic oscillators effectively masks the plaintext, preventing its retrieval by direct high-pass filtering. But plaintext signals of very low frequency may be still retrieved if we know what kind of non-linear time-varying system was used for encryption, but without the knowledge of its parameter and initial condition values. To show such a possibility we have implemented two cryptanalysis procedures based on generalized synchronization [24-27].

\subsection{Breaking the Lorenz system}

To break the PS-based chaotic masking scheme under study, using the Lorenz system, we use the following intruder receiver:

$$
\begin{aligned}
& \dot{x}_{2}=\sigma^{*}\left(y_{2}-x_{2}\right)+p \varepsilon, \\
& \dot{y}_{2}=\left(\mu^{*}-z\right) x_{2}-y_{2}+q \varepsilon .
\end{aligned}
$$

where $\left\{\sigma^{*}, \mu^{*}\right\}$ are the intruder receiver's Lorenz system parameters and $\varepsilon$ is the instantaneous value of the recovery error $\varepsilon=U(t)-\left(x_{2}+y_{2}\right)=m(t)+$ $x_{1}+y_{1}-x_{2}-y_{2}$. The terms $p \varepsilon$ and $q \varepsilon$ work as feedback of the recovery error, in order to achieve generalized synchronization between sender and receiver; $p$ and $q$ are two scalars that may accept a wide range of values, from 1 to more than 400, and even one of them alone may not exist. The synchronism is achieved for any combination of $p$ and $q$ values, but the amplitudes of $x_{2}$ and $y_{2}$ do not match with those of $x_{1}$ and $y_{1}$ while $\sigma^{*} \neq \sigma$ or $\mu^{*} \neq \mu$.

By making $p=\sigma^{*}$ Eq. (8) is simplified, becoming independent of $y_{2}$, hence not depending upon the adjustment of $\mu^{*}$. In this way $x_{1}=x_{2}$ whenever $\sigma^{*}=\sigma$, regardless of the value of $\mu^{*}$. Also we have found experimentally that the best results for fast convergence of the synchronism and minimum recovery error are obtained when $q=\sqrt{\sigma^{*}}$. With those settings our intruder receiver is redefined as:

$$
\begin{aligned}
& \dot{x}_{2}=-2 \sigma^{*} x_{2}+\sigma^{*} U(t) \\
& \dot{y}_{2}=\left(\mu^{*}-\sqrt{\sigma^{*}}-z\right) x_{2}-\left(1+\sqrt{\sigma^{*}}\right) y_{2}+\sigma^{*} U(t) .
\end{aligned}
$$

Figure 3 illustrates the synchronization mechanism between sender and intruder receiver. The values of the the sender parameters are $\{\sigma, \mu\}=\{10,60\}$ and the initial conditions of the sender and receiver are: $\left\{x_{1}(0), y_{1}(0), z_{1}(0), x_{2}(t), y_{2}(t)\right\}=$ 
(a) Sender; $\sigma=10, \mu=60$

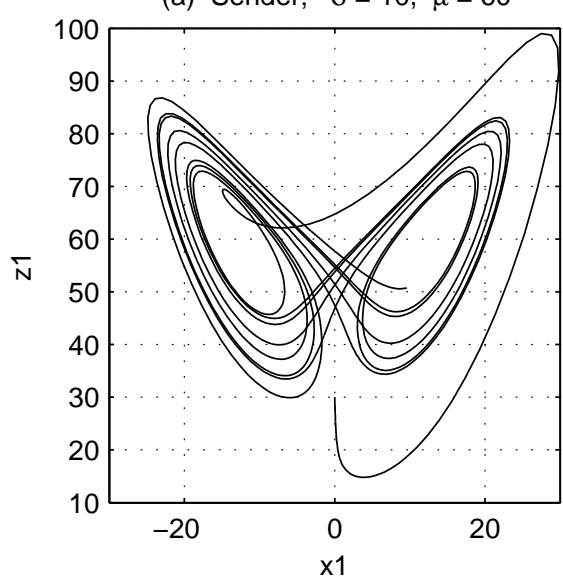

(d) $\sigma^{*}=10, \mu^{*}=80$

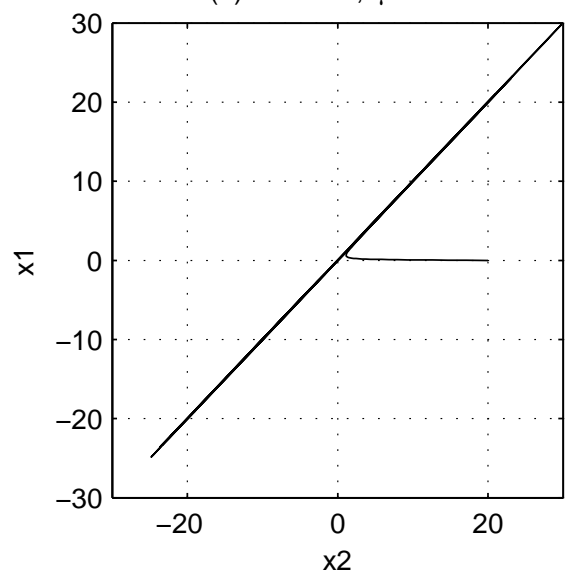

(b) $\sigma^{*}=10, \mu^{*}=80$

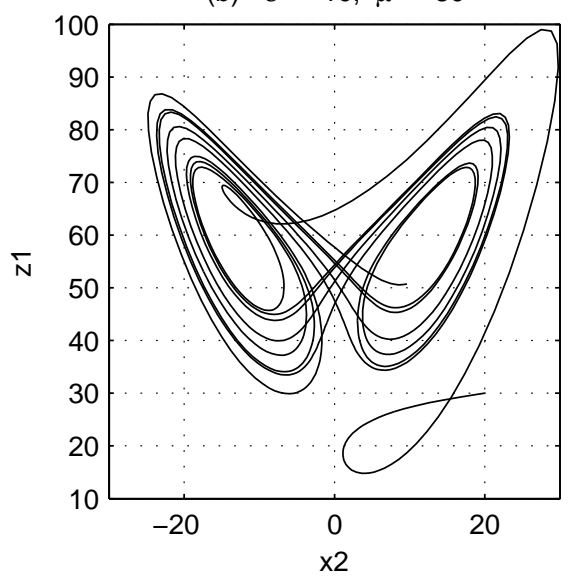

(c) $\sigma^{*}=40, \mu^{*}=80$

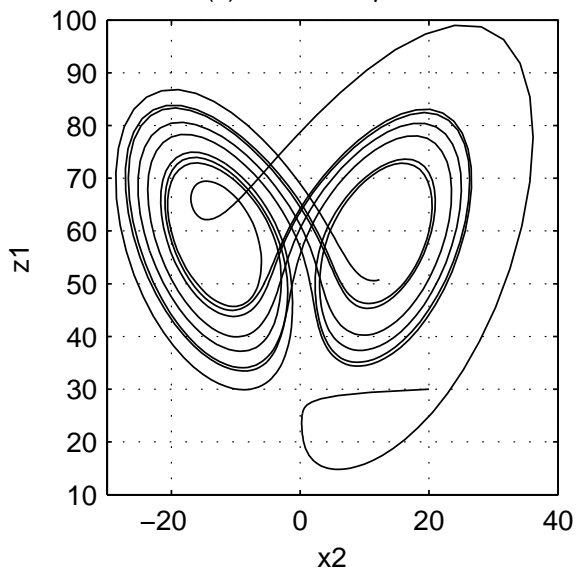

Fig. 3. Generalized synchronization of the Lorenz attractor: (a) plot of the sender variables $z_{1}$ vs. $x_{1}$, for $\{\sigma, \mu\}=\{10,60\}$; (b) plot of the sender variable $z_{1}$ vs. the intruder receiver variable $x_{2}$, for $\left\{\sigma^{*}, \mu^{*}\right\}=\{10,80\}$; (c) plot of the sender variable $x_{1}$ vs. the intruder receiver variable $x_{2}$, for $\left\{\sigma^{*}, \mu^{*}\right\}=\{10,80\}$; (d) plot of the sender variable $x_{1}$ vs. the intruder receiver variable $x_{2}$, when $\left\{\sigma^{*}, \mu^{*}\right\}=\{40,80\}$. The initial conditions in all cases were: $\left\{x_{1}(0), y_{1}(0), z_{1}(0), x_{2}(0), y_{2}(0)\right\}=\{0,0.2,30,20,1\}$.

$\{0,0.2,30,20,1\}$. Figure $3($ a) shows the first 8 seconds of the plot of the sender variables $z_{1}$ vs. $x_{1}$. Figure $3(\mathrm{~b})$ shows the plot of the sender variable $z_{1}$ vs. the intruder receiver variable $x_{2}$ when $\left\{\sigma^{*}, \mu^{*}\right\}=\{10,80\}$; comparing (a) and (b) it can be seen that both phase portraits are identical, after the short initial transient originated by the different initial conditions. Figure 3(c) shows the plot of the sender variable $x_{1}$ vs. the intruder receiver variable $x_{2}$, when $\left\{\sigma^{*}, \mu^{*}\right\}=\{10,80\}$; it can be seen that the phase and amplitude of $x_{2}$ match exactly those of $x_{1}$, after the initial transient, although $\mu^{*} \neq \mu$. Finally, Fig. 3(d) shows the plot of the sender variable $x_{1}$ vs. the intruder receiver variable $x_{2}$, when $\sigma^{*}$ and $\mu^{*}$ completely differ from $\sigma$ and $\mu$, revealing that both systems are synchronized, although their amplitudes and phases do not match exactly. 


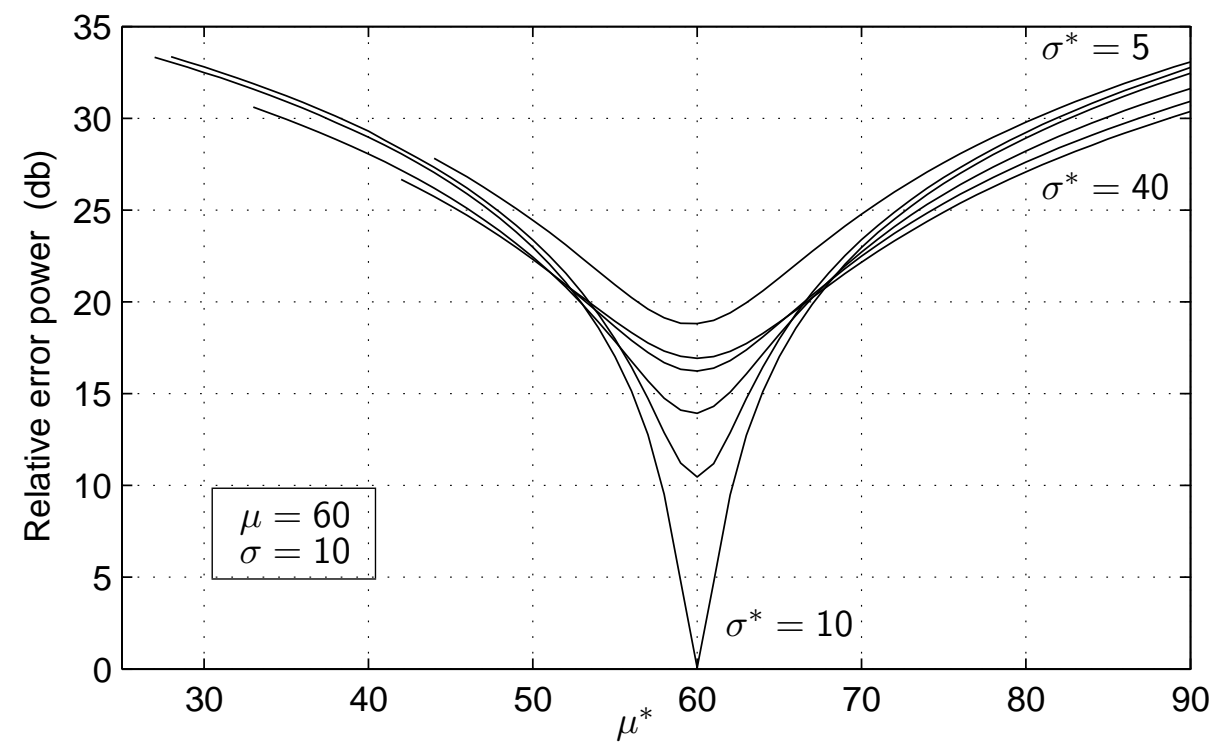

Fig. 4. Relative logarithmic representation of the mean of the error power $\varepsilon^{2}$, for $\sigma^{*}=\{5,7.5,10,20,30,40\}$ as a function of $\mu^{*}$.

We have estimated and recorded the logarithm of the mean value of the squared error $\varepsilon^{2}$, i.e. the error power, for the range of the intruder receiver system parameter values $\sigma^{*}$ and $r^{*}$ that give raise to the chaotic behavior of the Lorenz attractor, with the same transmitter system parameters of the numerical example presented in [19, Fig. 2] and the intruder receiver described by Eqs. (10) and (11). The results are presented in Fig. 4 . The mean of $\varepsilon^{2}$ is computed along the first 1.5 seconds, after a delay of 0.5 seconds to let the initial transient finish. It is clearly seen that the error grows monotonically with the mismatch between the transmitter and receiver parameters $\left\{\left|\sigma^{*}-\sigma\right|,\left|\mu^{*}-\mu\right|\right\}$, and that the minimum error corresponds to the receiver system parameters values $\left\{\sigma^{*}, \mu^{*}\right\}$ exactly matching the transmitter system parameters values $\{\sigma, \mu\}$.

The parameters value recovery procedure consists of the straightforward search for the minimum recovery error $\varepsilon$. Once the correct values $\left\{\sigma^{*}, \mu^{*}\right\}=\{\sigma, \mu\}$ are found, the term $x_{1}+y_{1}-x_{2}-y_{2}$ vanishes and the recovery error is just equal to the plaintext signal $m(t)$.

The search of the correct parameter values $\left\{\sigma^{*}, \mu^{*}\right\}$ can be done in the following way: first, select an initial value for $\sigma^{*}$ centered in its usable range; second, vary the value of $\mu^{*}$ until a minimum error is reached; third, keep this value and vary the value of $\sigma^{*}$ until a new minimum error is reached; four, check if the remaining error $\varepsilon$ is a clean recognizable plaintext, if not repeat the second and third steps. Note that this method retrieves all at once the correct values of $\sigma^{*}, \mu^{*}$, and the plaintext.

The procedure is illustrated in Fig. 5, for a plaintext $m(t)=\cos (2 \pi 4 t)$, whose frequency is so low that it cannot be retrieved by the previously described 

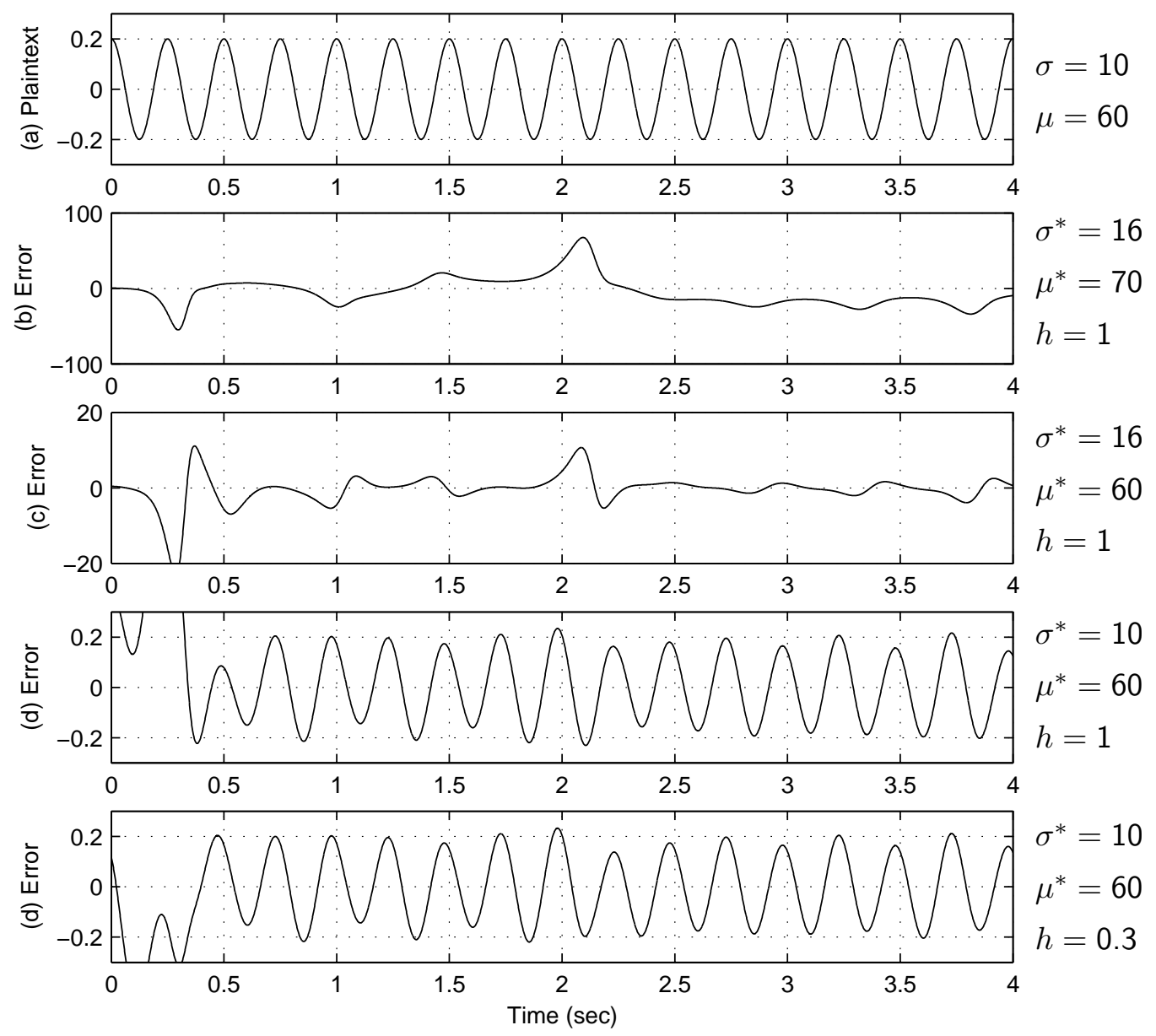

Fig. 5. Plaintext and parameter recovery of the Lorenz system by generalized synchronization analysis; the transmitter parameters are $\{\sigma, \mu, \rho\}=\{10,60,8 / 3\}$ : (a) plaintext $m(t)=\cos (2 \pi 4 t)$; (b) receiver error for unadjusted intruder receiver parameters $\left\{\sigma^{*}, \mu^{*}\right\}=\{16,70\}$; (c) receiver error for partially adjusted intruder receiver parameters $\left\{\sigma^{*}, \mu^{*}\right\}=\{16,60\}$; (d) receiver error for correct intruder receiver parameters $\left\{\sigma^{*}, \mu^{*}\right\}=\{10,60\}$; (e) receiver error for correct intruder receiver parameters $\left\{\sigma^{*}, \mu^{*}\right\}=\{10,60\}$, and unadjusted function $F=-0.7 x_{1}+0.3, y_{1}+m(t)$.

direct high-pass filtering method. When the initial parameter values are chosen at random as $\left\{\sigma^{*}, \mu^{*}\right\}=\{16,70\}$, the corresponding error reaches a peak value near 70 . Then the $\mu^{*}$ value is varied until a minimum of the error is found for $\mu^{*}=60$, it can be seen that now the peak error value after the initial transient is reduced to about 15 . Next, the $\sigma^{*}$ value is varied until a new error minimum is reached for $\sigma^{*}=10$, now the error is reduced to the plaintext itself, plus some noise of reduced amplitude that may be easily removed by a high-pass filter, if necessary.

We have found that our method woks as well for the whole family of functions of the form $F=(h-1) x_{1}+h y_{1}+m(t)$, were $h$ is a scalar of any value. The plaintext $m(t)$ is correctly recovered for any ciphertext of the form 
$U(t)=x_{1}+F\left[x_{1}, y_{1}, m(t)\right]$. This fact demonstrates that a great care must be exercised when selecting the function $F$, because some changes in its factors and constants values may be useless to enlarge the key space, hence not improving the system security at all. In Fig. 5(e) a time story of the retrieved message for a function of the form $F=-0.7 x_{1}+0.3, y_{1}+m(t)$ is presented, when decoded by an intruder receiver adjusted to recover a function of the type $F=y_{1}+m(t)$. It can be observed that the only difference with Fig. 5(d) is the magnitude and duration of the initial transient.

\subsection{Breaking the hyperchaotic Rössler system}

To break the PS-based chaotic masking scheme under study, when the hyperchaotic Rössler system is used to generate the masking signal, we follow a similar procedure to the one used in the preceding section. Now the intruder receiver is:

$$
\begin{aligned}
& \dot{x_{2}}=-y_{2}-z_{2}+p \varepsilon, \\
& \dot{y_{2}}=x_{2}+a y_{2}+w_{1}, \\
& \dot{z_{2}}=b+x_{2} z_{2} .
\end{aligned}
$$

were $p$ is a scalar. We have found the best results with $p=10$.

Here the instantaneous value of the recovery error is defined as $\varepsilon=U(t)-$ $\left(x_{2}+y_{2}\right)=m(t)+x_{1}+y_{1}-x_{2}-y_{2}$. When the synchronism is reached it happens that $x_{1}+y_{1}=x_{2}+y_{2}$, hence the error is $\varepsilon=m(t)$, thus allowing the exact recovery of the plaintext.

Figure 6 illustrates the plaintext recovered for various intruder receiver parameter values sets, being the plaintext $m(t)=\cos (2 \pi 2.5 t)$, whose frequency is so low that it cannot be retrieved by the previously described direct highpass filtering method. It can be observed that the intruder receiver is quite insensitive to the values of the parameters $\left\{a^{*}, b^{*}\right\}$ and the structure of the function $F$. In Fig. 6(b) it is shown that when the parameter values of sender and intruder receiver do not match at all, the plaintext is still visible, although a residual interference is present, but its intensity is not big enough to preserve the confidentiality of the communications. This interference can be easily removed, if desired, by trial and error in few steps. The first parameter to be adjusted is $a^{*}$, because it is the most influent on the shape of the retrieved waveform. In Fig. 6(c) it is shown the waveform for $a^{*}$ correctly adjusted while $b^{*}$ is kept unadjusted. Then the parameter $b^{*}$ must be adjusted until a clean recovered plaintext waveform is reached, as illustrated in Fig. 6(d).

We have tested several different invertible functions $F$ as building blocks of the sender ciphertext, and it has been observed that quite different functions allow 

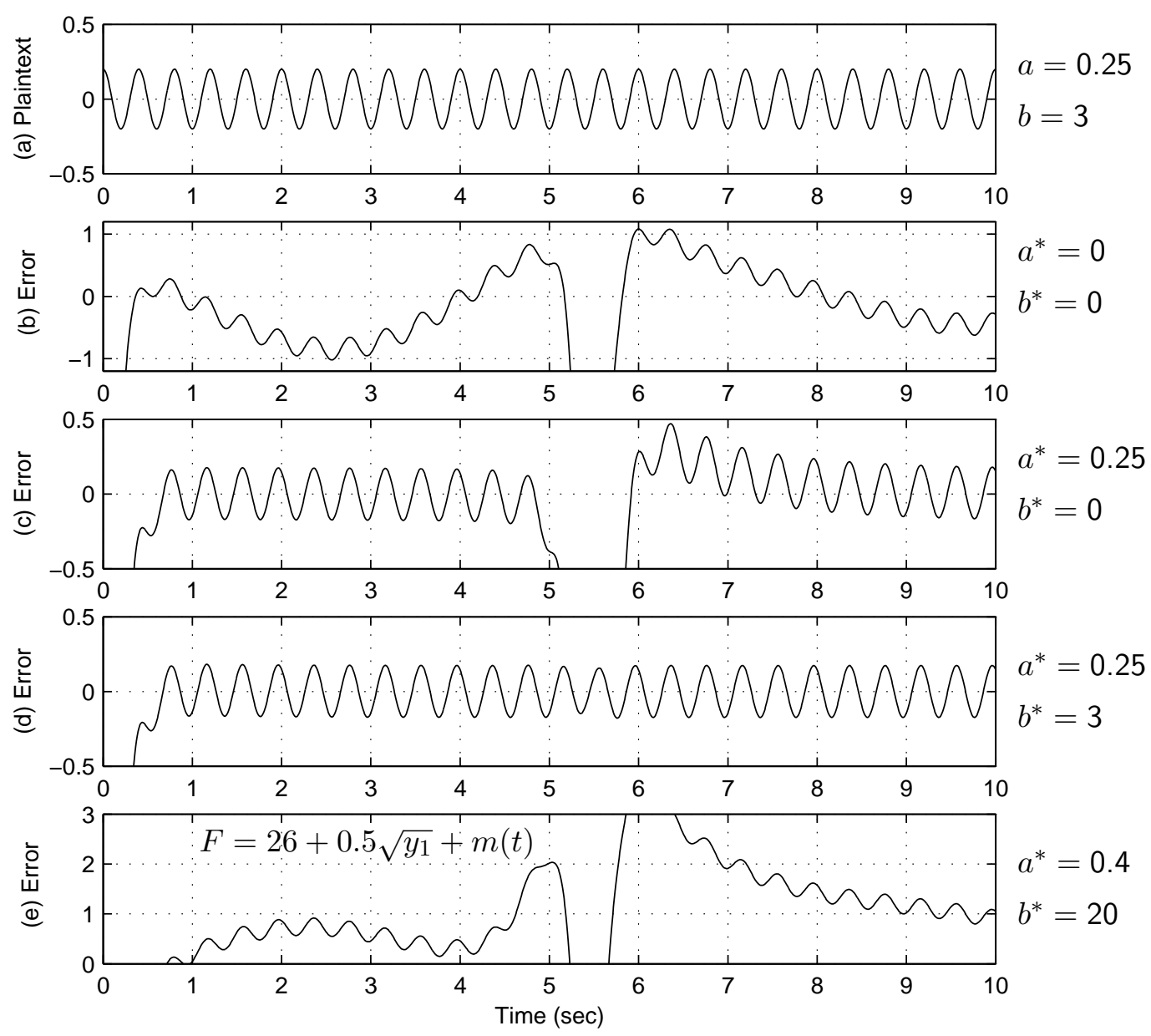

Fig. 6. Plaintext and parameter recovery by generalized synchronization analysis; the sender parameters are $\{a, b, c, d\}=\{0.25,3,-0.5,0.05\}$ : (a) plaintext $m(t)=\cos (2 \pi 2.5 t)$; (b) receiver error for unadjusted intruder receiver parameters $\left\{a^{*}, b^{*}\right\}=\{0,0\}$; (c) receiver error for partially adjusted intruder receiver parameters $\left\{a^{*}, b^{*}\right\}=\{0.25,0\}$; (d) receiver error for the correct intruder parameter values $\left\{a^{*}, b^{*}\right\}=\{0.25,3\}$. (e) receiver error for wrong intruder receiver parameter values $\left\{a^{*}, b^{*}\right\}=\{0.25,3\}$, and unadjusted function $F=26+0.5 \sqrt{y_{1}}+\cos (2 \pi 2.5 t)$.

for the almost correct retrieving of the plaintext. Figure 6(e) dramatically illustrates this fact: when a function as complicated as $F=26+0.5 \sqrt{y_{1}}+m(t)$ is used for transmission, while maintaining an intruder receiver designed for decoding a function of the type $F=y_{1}+m(t)$, together with a total parameter mismatch between sender and receiver as $\left\{a, b, a^{*}, b^{*}\right\}=\{0.25,3,0.4,20\}$, we can see that the retrieved waveform still retain enough plaintext information to completely compromise the security of the communication. 


\section{Other weaknesses of the proposed system}

The knowledge of the scaling factor $\alpha$ is not required to retrieve the plaintext, if a high-pass filter attack or a generalized synchronization recovering procedure are implemented, as we did. Hence the scaling factor does not add any strength to the system security, as opposed to the claims by the authors of [19].

Thanks to the plain transmission of the shared scalar variables $z_{1}$, or $w_{1}$, inherent to the PS scheme, the third Lorenz system parameter $\rho$ and the hyperchaotic Rössler parameters $c$ and $d$ need not be determined to recover the plaintext when using a generalized synchronization receiver, in opposition to other cryptosystems that make use the Lorenz or hyperchaotic Rössler systems as a masking signal. Therefore an additional advantage is offered by the authors of [19] to the opponent eavesdropper.

\section{Conclusion}

In summary, the proposed PS-based chaotic masking cryptosystem is rather weak, since it can be broken in two easy ways, the first one being ignorant of the transmitter precise structure and the second one knowing the transmitter structure but ignoring its parameter values. The alleged security advantage of the cryptosystem based on the eavesdropper lack of knowledge of the scaling factor $\alpha$ is incorrect, its knowledge is completely irrelevant to retrieve the plaintext. The function $F$ does not clearly enhance the security, owing to the fact that a wide range of different functions generate ciphertexts equally breakable with the same intruder receiver with a unique parameter adjustment. There is no mention about what the key is, nor which is the key space, a fundamental aspect in every secure communication system. The total lack of security discourages the use of this communication scheme for secure applications, unless some modifications are made to essentially enhance its security.

\section{Acknowledgements}

This work was supported by Ministerio de Ciencia y Tecnología of Spain, research grants TIC2001-0586 and SEG2004-02418, and by the Applied R\&D Center, City University of Hong Kong, Hong Kong SAR, China, under Grants no. 9410011 and no. 9620004 . 


\section{References}

[1] L. M. Pecora, T. L. Carroll, Synchronization in chaotic systems, Phys. Rev. Lett. 64 (8) (1990) 821-824.

[2] L. M. Pecora, T. L. Carroll, Driving systems with chaotic signals, Phys. Rev. A 44 (4) (1991) 2374-2383.

[3] T. L. Carroll, L. M. Pecora, Synchronizing chaotic circuits, IEEE Trans. Circ. Syst. 38 (4) (1991) 453-456.

[4] T. Yang, A survey of chaotic secure communication systems, Int. J. Comput. Cognition 2 (2004) 81-130.

[5] K. M. Cuomo, A. V. Oppenheim, Chaotic signals and systems for communications, in: Proc. IEEE ICASSP III, Vol. III, 1993, pp. 137-140.

[6] K. M. Cuomo, A. V. Oppenheim, Circuit implementation of synchronized chaos with applications to communications, Phys. Rev. Lett. 71 (1) (1993) 65-68.

[7] C. W. Wu, L. O. Chua, A simple way to synchronize chaotic systems with applications to secure communication systems, Int. J. Bifurcation Chaos 3 (6) (1993) 1619-1627.

[8] R. Lozi, L. O. Chua, Secure communications via chaotic synchronization. II. noise reduction by cascading two identical receivers, Int. J. Bifurcation Chaos 3 (5) (1993) 1319-1325.

[9] S. J. Li, X. Q. Mou, Y. L. Cai, Improving security of a chaotic encryption approach, Phys. Lett. A 290 (3-4) (2001) 127-133.

[10] K. M. Short, Steps toward unmasking secure communications, Int. J. Bifurcation Chaos 4 (4) (1994) 959-977.

[11] C. S. Zhou, T. L. Chen, Extracting information masked by chaos and contaminated with noise: Some considerations on the security of communication approaches using chaos, Phys. Lett. A 234 (6) (1997) 429-435.

[12] G. Álvarez, F. Montoya, M. Romera, G. Pastor, Cryptanalysis of a chaotic secure communication system, Phys. Lett. A 306 (4) (2003) 200-205.

[13] G. Álvarez, F. Montoya, M. Romera, G. Pastor, Cryptanalysis of a discrete chaotic cryptosystem using external key, Phys. Lett. A 319 (3-4) (2003) 334339 .

[14] S. Li, X. Mou, Y. Cai, Z. Ji, J. Zhang, On the securrity of a chaotic encryption scheme: problems with computerized chaos in finite computing precision, Comput. Phys. Commun. 153 (1) (2003) 52-58.

[15] S. Li, X. Mou, B. L. Yang, Z. Ji, J. Zhang, Problems with a probabilistic encryption scheme based on chaotic systems, Int. J. Bifurcation Chaos 13 (10) (2003) 3063-3077. 
[16] G. Álvarez, F. Montoya, M. Romera, G. Pastor, Breaking parameter modulated chaotic secure communication system, Chaos Solitons Fractals 156 (4) (2004) $783-787$.

[17] R. Mainieri, J. Rehacek, Projective synchronization in three-dimensional chaotic systems, Phys. Rev. Lett. 82 (15) (1999) 3042-3045.

[18] D.Xu, Z. Li, Controlled projective synchronization in nonparametrically-linear chaotic systems, Int. J. Bifurcation Chaos 12 (6) (2002) 1395-1402.

[19] Z. Li, D. Xu, A secure communication scheme using projective chaos synchronization, Chaos Solitons Fractals 22 (2) (2004) 477-481.

[20] D. R. Stinson, Cryptography: theory and practice, CRC Press, Boca Raton, 1995.

[21] R. L. Devaney, A first course in chaotic dynamical systems, Addison-Wesley, Reading, MA., 1992.

[22] H. L. Oson, Music, Physics and Engineering, 2nd Edition, Dover, New York, 1967.

[23] J. M. Eargle, Music, Sound and Technology, Van Nostrand Reinhold, New York, 1990.

[24] N. F. Rulkov, M. M. Sushchik, L. S. Tsimring, H. D. I. Abarbanel, Generalized synchronization of chaos in directionally coupled chaotic systems, Phys. Rev. E 51 (2) (1995) 980-994.

[25] L. Kocarev, U. Parlitz, Generalized synchronization, predictability, and equivalence of unidirectionally coupled dynamical systems, Phys. Rev. Lett. 76 (11) (1996) 1816-1819.

[26] T. Yang, L.-B. Yang, C.-M. Yang, Breaking chaotic switching using generalized synchronization: examples, IEEE Trans. Circuits Syst. I 45 (10) (1998) 10621067 .

[27] G. Álvarez, L. Hernández, F. Montoya, J. Muñoz, Cryptanalysis of a novel cryptosystem based on chaotic oscillators and feedback inversion, J. Sound Vibrat. 275 (1-2) (2004) 423-430. 